\title{
Deprivation state determines the motivational effects of neuroleptics in rats
}

\author{
FRANCES HARRINGTON and DEREK VAN DER KOOY \\ University of Toronto, Toronto, Ontario, Canada
}

\begin{abstract}
The role of food-deprivation states in determining the motivational effects of the neuroleptic alpha-flupenthixol was investigated using a place conditioning paradigm. During testing, rats preferred an environment that was previously paired with food deprivation and alpha-flupenthixol pretreatment $(0.8 \mathrm{mg} / \mathrm{kg} / \mathrm{ml}$, i.p.) over an environment that was previously paired with food deprivation and saline pretreatment. Indeed, alpha-flupenthixol blocked the conditioned place aversions produced by hunger in food-deprived animals, but had no motivational effects in nondeprived animals. We suggest that dopaminergic neurons provide a motivational substrate only when animals are in a state of deprivation.
\end{abstract}

Evidence from lesion and pharmacological studies suggests a critical involvement of dopaminergic neurons in the rewarding properties of psychostimulants (Mackey \& van der Kooy, 1985; Mithani, Martin-Iverson, Phillips, \& Fibiger, 1986; Spyraki, Fibiger, \& Phillips, 1982b; Yokel \& Wise, 1976), food (Evans \& Vaccarino, 1990; Spyraki, Fibiger, \& Phillips, 1982a; Wise \& Raptis, 1986), and opiates (Bozarth \& Wise, 1981; Shippenberg \& Herz, 1987; Hand, Stinus, \& LeMoal, 1989). In an attempt to account for the common element among these types of effects, Wise, Spindler, DeWit, and Gerber (1978) proposed the anhedonia hypothesis, which states that the administration of dopamine blockers blunts the hedonic impact of all rewarding stimuli. Standing in opposition to this general anhedonia hypothesis are a number of studies that fail to demonstrate neuroleptic blockade of either food (Bechara, Harrington, Nader, \& van der Kooy, 1992; Irwin, Tombaugh, Zacharko, \& Anisman, 1983) or opiate reward (Ettenberg, Pettit, Bloom, \& Koob, 1982; Mackey \& van der Kooy, 1985). It is worth noting here that neuroleptics also produce numerous behavioral effects that are not easily described as products of reward or motivational processes (see Salamone, 1992).

We suggest that the conflicting motivational results might be resolved if dopamine blockade interferes with only a subclass of rewarding events, such as those that are dependent on an existent state of deprivation. Indeed, we have employed specific brain manipulations to double dissociate the rewarding effects of both opiates and food in nondeprived versus deprived animals. Lesions of the tegmental pedunculopontine nucleus (TPP) block the re-

This research was supported by the Medical Research Council of Canada. We thank Tony Bechara for helpful advice. Address correspondence to D. van der Kooy, Department of Anatomy and Cell Biology, Faculty of Medicine, University of Toronto, Toronto, Ontario, Canada M5S 1A8. warding effects of food in food-sated rats and of opiates in previously drug-naive rats (Bechara \& van der Kooy, 1989, 1992). TPP lesions fail to block the rewarding effects of food in food-deprived rats and the rewarding effects of morphine in opiate-dependent and opiatewithdrawn rats (Bechara \& van der Kooy, 1992). On the other hand, neuruoleptics do not block the rewarding effects of opiates in opiate-naive animals nor of food in foodsated animals, but do block the rewarding effects of opiates in opiate-dependent and opiate-withdrawn animals and the aversiveness of hunger in food-deprived rats (Bechara, Harrington, Nader, \& van der Kooy, 1992). When animals are in a food-deprived state, neuroleptics also block food reward (Spyraki et al., 1982a; Wise et al., 1978). We therefore hypothesize that the ability of neuroleptics to disrupt reward is wholly dependent upon the motivational state of the animal at the time of neuroleptic administration.

The general anhedonia hypothesis implies that dopamine blockade by neuroleptics in itself should produce (in the absence of other explicit rewarding stimuli) aversions not present in the normal nonneuroleptic state. In fact, the data on this point appear more complicated than predicted. In different studies, the administration of neuroleptics induced motivational states that were rewarding (Glick \& Cox, 1975; Hoffman \& Beninger, 1989), aversive (Shippenberg \& Herz, 1987, 1988), or motivationally neutral (Bozarth \& Wise, 1981; Hoffman \& Beninger, 1989; Mackey \& van der Kooy, 1985). Our previous data demonstrating that the ability of neuroleptics to block the rewarding impact of various stimuli depends on deprivation state (Bechara et al., 1992; Bechara \& van der Kooy, 1992) led to our current hypothesis that changing the deprivation state of rats should change the motivational states produced by neuroleptics themselves. The three experiments in the present report tested this hypothesis. In the first experiment, we looked at the baseline motivational effects of neuroleptics in a place conditioning paradigm at different times after neuroleptic administration. In Ex- 
periment 2 , we asked if food deprivation modified the motivational effects of neuroleptics, and, in Experiment 3, if the motivational effects of food deprivation were blocked by neuroleptics.

\section{EXPERIMENT 1}

Neuroleptics, by themselves, have been shown to produce varying motivational states. In the place conditioning paradigm, Mackey and van der Kooy (1985) have previously demonstrated that administration of alphaflupenthixol $2.5 \mathrm{~h}$ prior to place conditioning produced neither preferences for nor aversions to the drugconditioned environment. Other studies, using lower doses of alpha-flupenthixol, have suggested that some of the maximal behavioral effects of the drug are seen $4 \mathrm{~h}$ after injection (Corbett, Stellar, Stinus, Kelley, \& Fouriezos, 1983). Experiment 1 examined the baseline motivational properties of environments associated with neuroleptic action 2.5 and $4 \mathrm{~h}$ after drug administration.

\section{Method}

Subjects. The animals used in Experiments 1-3 were adult male Wistar rats (Charles River), weighing between $300-400 \mathrm{~g}$. The subjects were housed individually in suspended gray wires cages, in a room kept at a temperature of $22^{\circ} \mathrm{C}$ and illuminated between 0900-2100 h. Water was continuously available throughout the experiments. Purina Rat Chow was also available ad lib except during the food-deprivation experiments, when it was available only for restricted periods as detailed below.

Apparatus. A place conditioning paradigm was used in all three experiments to determine motivational effects, because this paradigm has been shown to be a simple and effective method for assessing the motivational properties of various stimuli (van der Kooy, 1987). The place conditioning apparatus was identical to that described in Mucha, van der Kooy, O'Shaughnessy, and Bucenieks (1982). Prior to conditioning, the animals were handled and exposed to a gray box in order to familiarize them with the handling techniques of the experimenter and to an environment grossly similar to those in which they would be conditioned. Conditioning took place in one of two boxes, which differed in color, texture, and smell. One box had black walls and a black Plexiglas floor that was wiped with a $2 \%$ acetic acid solution just prior to use. The other box had white walls and a mesh floor covered with wood chips, which gave it a slight smell of wood. Conditioning involved pairing one of these specific environments with a rewarding stimulus or a motivational state, and the other environment with the absence of that stimulus or the presence of a different motivational state. Our conditioning procedure involved pairing each environment four times (45 min for each pairing) with its appropriate stimulus or state. The order of injections and the order of the presentation of the paired environments were counterbalanced within groups.

During testing, a narrow, neutral gray zone separated the two conditioning compartments. On the test day, each rat was placed in the neutral gray zone in a drug-free, nondeprived state and was allowed to freely explore each previously conditioned environment for a period of $10 \mathrm{~min}$. The time spent in each compartment was recorded by an observer using an electric chronometer. All place conditioning testing was carried out with the observer being blind as to which box was associated with which motivational stimulus or state. For scoring purposes, the rat was defined as being in a given compartment when both forepaws were located in that compartment. This place conditioning apparatus, which balances the baseline preferences for the two compartments, has repeatedly been shown to yield equal preferences for both environments when nonconditioned animals are allowed to freely explore both environments (Mucha \& Iversen, 1984; Mucha et al., 1982). Furthermore, even when uninjected or saline-injected (vehicle) naive rats were repeatedly placed in only one environment of a place conditioning apparatus, they showed equal preferences for the familiar environment and the unfamiliar neutral environment (Bechara et al., 1992; Bechara \& van der Kooy, 1989; Mucha \& Iversen, 1984).

Procedure. Separate groups of food-sated rats, pretreated $2.5 \mathrm{~h}$ prior to conditioning ( $n=8)$ or 4 h prior to conditioning $(n=16)$, were employed. Pretreatment consisted of the administration of vehicle (saline) or drug (alpha-flupenthixol) prior to conditioning. The presence of the neuroleptic alpha-flupenthixol was paired with one environment and the saline vehicle with the other environment. Over 8 days, the animals were injected intraperitoneally (i.p.) with a $0.9 \%$ physiologic saline solution on 4 days, and with a $0.8-\mathrm{mg} / \mathrm{kg} / \mathrm{ml} \mathrm{so-}$ lution of alpha-flupenthixol on the 4 alternate days, and then placed in the appropriate distinct environment. Alpha-flupenthixol is a broad-spectrum dopamine antagonist that binds with at least a minor selectivity to the D1 dopamine receptor (Seeman, 1980), but that at the high dose used here probably binds relatively nonselectively to both the D1 and D2 receptor subtypes (Enna, Bennett, Burt, Creese, \& Snyder, 1976). Testing occurred 2 days after the last conditioning trial.

\section{Results and Discussion}

No place preferences or aversions were evident in the group that was administered the neuroleptic $2.5 \mathrm{~h}$ prior to conditioning, but clear conditioned place aversions were evident with the administration of the neuroleptic $4 \mathrm{~h}$ prior to conditioning (Figure 1). An analysis of variance (ANOVA) on these data revealed a significant interaction between group and times spent on the alpha-flupenthixolversus saline-paired sides $[F(1,22)=6.38, p<.05]$. Thus, the times that the rats spent on the two sides of the test apparatus depended upon when they received alphaflupenthixol prior to conditioning. The 2.5 -h pretreatment rats did not spend significantly different amounts of time in the saline- and alpha-flupenthixol-paired environments $[t(7)=0.09, p>.10]$. The 4-h pretreatment rats spent significantly less time in the alpha-flupenthixol-paired environment than in the saline-paired environment $[t(15)=$ $5.50, p<.05]$.

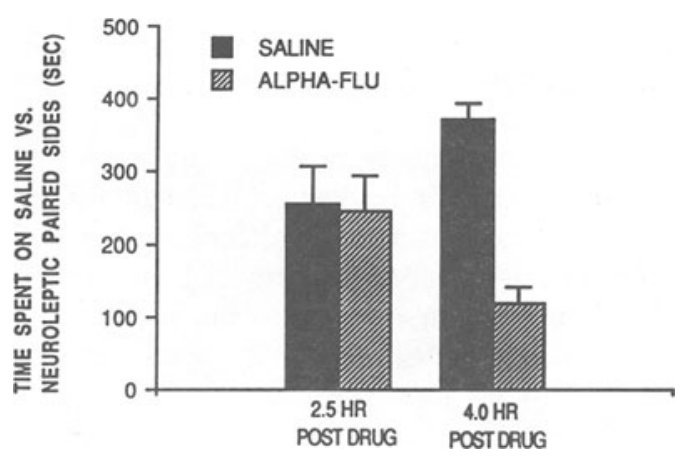

Figure 1. Effects of varying the interval between drug administration and place conditioning. Data represent means \pm SEM in both the 2.5-h group $(n=8)$ and in the 4.0-h group $(n=16)$. 
These results concur with those of Mackey and van der Kooy (1985), showing that alpha-flupenthixol induces no motivational state of its own when administered $2.5 \mathrm{~h}$ prior to conditioning. The reason that this same neuroleptic induced aversions $4 \mathrm{~h}$ after its administration remains unclear. Neuroleptics were certainly behaviorally effective at both 2.5 and $4 \mathrm{~h}$ after administration, because the rats were completely cataleptic in the conditioning boxes, compared with their normal exploratory behavior in the conditioning box on saline vehicle days. It seems conceivable that the longer behavioral catelepsy persists, the more aversive it becomes, and thus, conditioning $4 \mathrm{~h}$ after alpha-flupenthixol would reveal greater aversions. In a similar vein, perhaps the rats were associating the alphaflupenthixol environment with hunger in the case of administration $4 \mathrm{~h}$ prior to conditioning, but not in the case of administration $2.5 \mathrm{~h}$ prior to conditioning. Administering high doses of neuroleptics depresses feeding in rats (Rowland \& Engle, 1977; Wise \& Raptis, 1986). When alpha-flupenthixol was administered $2.5 \mathrm{~h}$ prior to conditioning, the rats may have fasted for only a short period and were not hungry during the conditioning period. In contrast, when alpha-flupenthixol was administered $4 \mathrm{~h}$ prior to conditioning, the rats had fasted for a longer period of $4 \mathrm{~h}$ and may have been hungry during the conditioning period. We have previously shown that rats show conditioned aversions for environments paired with food deprivation, compared with novel, neutral environments (Bechara et al., 1992; Bechara \& van der Kooy, 1992). Nevertheless, this explanation seems inconsistent with our previous finding (Bechara et al., in press) that neuroleptics block the aversiveness of food deprivation (see also Experiment 3, the present study). One would have to suggest that by $4 \mathrm{~h}$ and later after alpha-flupenthixol administration, levels of neuroleptic will have fallen sufficiently so as to no longer block the aversiveness of hunger, but not far enough to permit all the motoric actions involved in feeding behavior. Similar reasoning would suggest that alpha-flupenthixol administered $2.5 \mathrm{~h}$ prior to conditioning would continue to block the aversiveness of any hunger that developed for long enough after removal from the environment to prevent any forward conditioning from occurring. This hypothesis could be tested by feeding the rats with food, possibly by intubation, immediately prior to all conditioning trials, and then seeing if this feeding blocks the aversiveness of alpha-flupenthixol given $4 \mathrm{~h}$ prior to conditioning.

Regardless of the explanation for the aversive effects that developed $4 \mathrm{~h}$ after alpha-flupenthixol administration, the lack of significant motivational effects seen $2.5 \mathrm{~h}$ after alpha-flupenthixol administration provided a neutral baseline motivational response that we could attempt to manipulate into approach or avoidance responses by modulation of deprivation state.

\section{EXPERIMENT 2}

In Experiment 2, we examined how the motivational state of the rats (food deprived vs. food sated) altered the motivational properties of alpha-flupenthixol. We predicted that if dopamine plays a motivational role in rats in a deprived state, but not in rats in a sated state (Bechara et al., 1992), then dopamine blockers would only produce motivational effects in food-deprived rats. Thus, we tested for conditioned preferences or aversions for alphaflupenthixol- versus saline-paired environments in foodsated and in food-deprived groups.

\section{Method}

Procedure. Separate groups of food-sated $(n=8)$ and fooddeprived $(n=8)$ rats were place conditioned such that the neuroleptic alpha-flupenthixol was paired with one environment and the saline vehicle with the other. Each of the rats in the food-sated and food-deprived groups received either an i.p. injection of the $0.9 \%$ physiologic saline solution (vehicle) or a $0.8-\mathrm{mg} / \mathrm{kg} / \mathrm{ml}$ solution of alpha-flupenthixol (drug) $2.5 \mathrm{~h}$ prior to conditioning in distinct environments. Four vehicle and four drug conditioning trials were alternated (one every 2 days) over 16 days. Approximately $5 \mathrm{~h}$ following return to their home cages after each conditioning trial, the food-deprived rats received 1-h ad-lib access to Purina Rat Chow in order to maintain body weight, and then were not fed again until the following day. To ensure a constant $22 \mathrm{~h}$ of food deprivation at the time the animals in the food-deprived group were in the conditioning environments, the conditioning trials were run every 2 nd day, over the period of 16 days. On the nonconditioning days, the food-deprived rats received only the normal 1-h access to food ad lib. At the termination of this feeding period, the 22-h fooddeprivation period commenced. The food-deprived rats consumed an average of $10 \mathrm{~g}$ of Purina Rat Chow each day while in the home cage during the 1-h food-access period. The food-sated rats had ad-lib access to food when in their home cages (and consumed an average of approximately $30 \mathrm{~g}$ of Purina Rat Chow per day during the experiment). In both groups of rats, food was never presented in the conditioning boxes. Other aspects of place conditioning and testing were identical to those described in Experiment 1. All the rats were tested in a food-sated, drug-free state, 5 days after the end of the conditioning trials.

\section{Results and Discussion}

No conditioned place preferences or aversions to the alpha-flupenthixol-paired environment were seen in the food-sated group, however, there were conditioned place preferences for the alpha-flupenthixol-paired environment in the food-deprived group (Figure 2). An ANOVA on these data revealed a significant interaction between

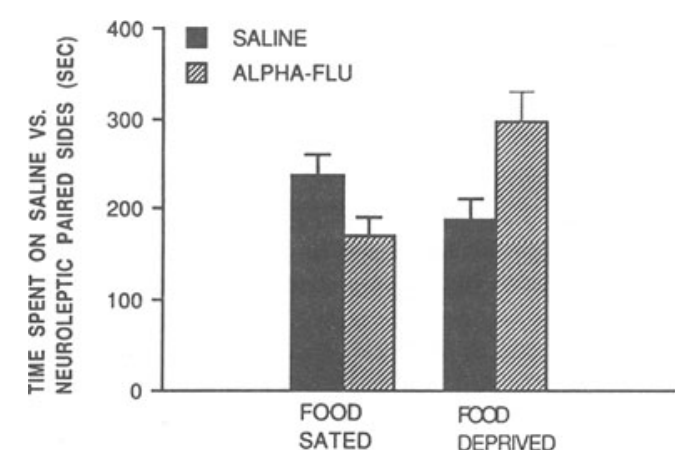

Figure 2. Place conditioning with neuroleptics in food-sated and food-deprived rats. Data represent means $\pm S E M$ for 8 rats. 
groups and the times spent on the alpha-flupenthixolversus saline-paired sides $[F(1,14)=8.28, p<.05]$. Thus, the times the rats spent on the alpha-flupenthixolversus saline-paired sides of the test apparatus depended on their motivational state (food deprived or food sated). The subjects in the food-sated group did not spend significantly different amounts of time in the saline- and the neuroleptic-paired environments $[t(7)=1.84, p>.10]$. However, the subjects in the food-deprived group spent more time in the neuroleptic-paired environment $[t(7)=$ $2.21, p<.06]$

In contrast to the implication of the general anhedonia hypothesis that dopamine blockade should be aversive or dysphoric, the present experiment reveals that neuroleptics can actually approach responses in the place conditioning paradigm under certain conditions. We suggest that a sufficient condition is training the animals in a state of food deprivation. We hypothesize that, at the time of the place conditioning test, the animals were faced with a choice between an environment signaling the aversiveness of food deprivation and an alternative environment signaling the neuroleptic-induced alleviation of the aversiveness of food deprivation. Thus, the previously fooddeprived animals show a conditioned approach response to an environment paired with alpha-flupenthixol. This proposed explanation for the results assumes that alphaflupenthixol blocked the aversiveness of food deprivation. This assumption was tested directly in Experiment 3.

\section{EXPERIMENT 3}

If the conditioned approach responses to alpha-flupenthixol in food-deprived rats are due to the neuroleptic's blockade of the aversiveness of hunger, then the neuroleptic should abolish the motivational difference between the food-sated and the food-deprived states. Experiment 3 was undertaken to determine if the aversive motivational effects of the food-deprived state (compared with the foodsated state) were blocked by the administration of the neuroleptic alpha-flupenthixol. Two groups, one given a saline injection prior to all conditioning trials and the other given alpha-flupenthixol prior to all conditioning trials, were each conditioned in the food-deprived state in one environment, and in the food-sated state in the alternate environment. We predicted that neuroleptics would block the acquisition of conditioned place aversions to an environment paired with food deprivation when compared with an environment paired with food satiation.

\footnotetext{
Method

Procedure. Separate groups of saline-pretreated $(n=8)$ and alphaflupenthixol-pretreated $(n=8)$ rats were place conditioned such that food satiation was paired with one environment and food deprivation with the other. The saline group was pretreated with an i.p. injection of a $0.9 \%$ physiologic saline solution $2.5 \mathrm{~h}$ prior to conditioning in both environments. Similarly, the neuroleptic group was pretreated with an i.p. injection of $0.8-\mathrm{mg} / \mathrm{kg} / \mathrm{ml}$ of the alphaflupenthixol $2.5 \mathrm{~h}$ prior to conditioning in both environments. Four food-sated conditioning trials were altemated with four food-deprived conditioning trials (one every 2 days) over 16 days. The rats in both
}

groups received food in their home cages approximately $5 \mathrm{~h}$ after each food-deprivation conditioning trial. From then until their next food-sated conditioning trial, all the animals had ad-lib access to food in their home cages. Prior to their food-deprived conditioning trials, the rats in both groups had ad-lib access to food until $22 \mathrm{~h}$ prior to conditioning, at which time the food was removed. In both groups of rats, food was never presented in the conditioning boxes. Other aspects of place conditioning and testing were identical to those described in Experiment 1. All the rats were tested in a food-sated, drug-free state, 3 days after the end of the conditioning trials.

\section{Results and Discussion}

Conditioned aversions for environments associated with food deprivation were blocked by the administration of alpha-flupenthixol (Figure 3). An ANOVA on these data revealed a significant interaction between the saline- and alpha-flupenthixol-pretreated groups and the times spent in the environment paired with food deprivation versus the environment paired with food satiation $[F(1,14)=9.49$, $p<.05]$. Thus, the times the rats spent on the sides of the test apparatus associated with different food-deprivation states depended on whether they had received the saline or the alpha-flupenthixol pretreatment. The subjects in the saline-pretreated group spent significantly less time in the environment associated with the state of food deprivation than in the environment associated with food satiation $[t(7)$ $=2.86, p<.05]$. However, the subjects in the alphaflupenthixol-pretreated group did not spend significantly different amounts of time in the previously food-sated and the previously food-deprived paired environments $[t(7)=$ $1.21, p>.10]$.

The results from this experiment reveal that food deprivation produces an aversive state, and that this aversiveness can be blocked by the administration of alphaflupenthixol. This result is consistent with our previous finding that alpha-flupenthixol blocks the conditioned avoidance of a hunger-paired environment, in a modified place conditioning paradigm where only one environment is paired with food deprivation and the other environment is novel at the time of testing (Bechara et al., 1992).

Neuroleptics can block both the aversiveness of a deprivation state and the rewarding properties of stimuli alleviating that state. Neuroleptics block both opiate reward

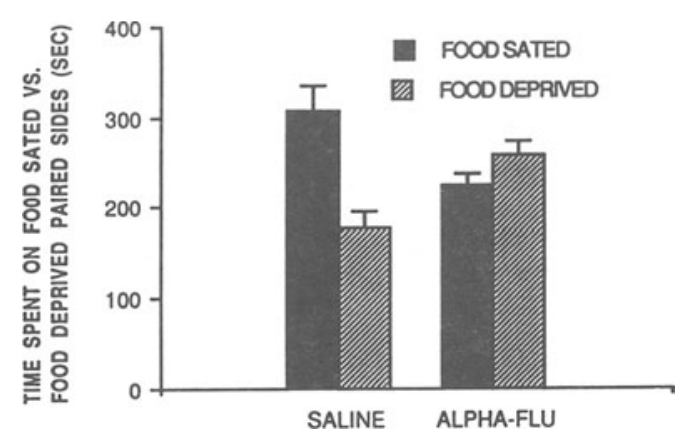

Figure 3. Effects of pretreatment with neuroleptics on the place aversions produced by food deprivation. Data represent means \pm SEM for 8 rats. 
in opiate-dependent and opiate-withdrawn animals and the aversiveness of opiate withdrawal in opiate-dependent animals (Bechara et al., 1992). Similarly, when animals are in a food-deprived state, neuroleptics block both food reward (Spyraki et al., 1982a; Wise et al., 1978) and the aversiveness of food deprivation (Bechara et al., 1992). In contrast, neuroleptics block neither food reward in sated rats, nor opiate reward in previously drug-naive rats (Bechara et al., 1992). Dopaminergic neurons must therefore be involved in motivational events that are activated when the animal is in a state of deprivation. More specifically, dopaminergic neurons must be involved in both the aversive mechanism of deprivation and a reward mechanism associated with the relief of this aversive state. It remains to be explained how dopamine acts in this dual capacity, but several possibilities exist. First, a subtle change in the levels of dopamine may determine aversion or reward while in the deprived state. Perhaps, neuroleptics so completely block dopaminergic neurons that no changes in dopamine levels can be detected and the motivational state becomes neutral. A second explanation suggests that perhaps there are two separate dopaminergic subsystems mediating these opposite motivational effects (the aversiveness of food deprivation and the relief from this deprivation) in deprived animals. A third explanation suggests that other neural systems also may be involved in the aversiveness of deprivation, and neuroleptics could be interfering with these. At the doses we used in this experiment, alpha-flupenthixol may nonselectively bind to noradrenergic as well as to dopaminergic receptors (Blumberg, Taylor, \& Sulser, 1975; Bowman \& Rand, 1980), and there is evidence for noradrenergic involvement in the effects of drug deprivation (Rasmussen, Beitner-Johnson, Krystal, Aghajanian, \& Nestler, 1990; Redmond \& Krystal, 1984).

\section{GENERAL DISCUSSION}

The attractiveness of the anhedonia hypothesis (Wise et al., 1978) is derived from its suggestion that the hedonic or rewarding properties of a variety of stimuli could be blocked by neuroleptics, and thus the motivational properties of all these stimuli must be mediated by a single brain substrate (dopaminergic neurons). Two types of evidence have refuted the most general version of the anhedonia hypothesis. First, experimental data showed that under certain conditions, neuroleptics did not block the rewarding effects of food (Bechara, Harrington, Nader, \& van der Kooy, 1992; Irwin et al., 1983) and opiates (Ettenberg et al., 1982; Mackey \& van der Kony, 1985). Second, an implication of the anhedonia hypothesis has not been borne out-that neuroleptics themselves (in the absence of other explicit rewarding stimuli) should produce dysphoria or aversive states. Indeed, under different conditions the motivational properties of neuroleptics have been suggested to be aversive (Shippenberg \& Herz, 1987), neutral (Bozarth \& Wise, 1981; Hoffman \& Beninger, 1989; Mackey \& van der Kooy, 1985), or rewarding (Glick \&
Cox, 1975; Hoffman \& Beninger, 1989), all of which were found in the present results as well.

Both of these types of data standing against the general anhedonia hypothesis contain evidence that forms the seed for a new hypothesis of dopamine's role in motivation. First, some of the failures to observe neuroleptic blockade of rewarding stimuli seem to involve instances of animals trained in nondeprivation states. Neuroleptics fail to block the rewarding effects of morphine only in animals that were previously naive to opiates (Bechara et al., 1992), and thus not dependent on opiates and subject to drug withdrawal. Neuroleptics also failed to block the rewarding effects of food when animals were trained in a food-sated state (Bechara et al., 1992). Second, and most important, the present results demonstrate that the motivational properties of neuroleptics themselves can be switched from conditioned approach to conditioned avoidance simply by manipulating the food-deprivation state of animals during place conditioning. Alpha-flupenthixol produces strong behavioral effects (catelepsy) $2.5 \mathrm{~h}$ after its systemic administration, but appears to have neutral motivational properties (Experiment 1). However, $4 \mathrm{~h}$ after its administration, alpha-flupenthixol produces aversive effects, possibly due to its higher cumulative activity at this time (Experiment 1). On a baseline of food deprivation, alpha-flupenthixol produces conditioned approach responses $2.5 \mathrm{~h}$ after its systemic administration, because it blocks the aversive effects of hunger when compared with the saline-pretreated aversiveness of hunger (Experiment 2). In this light, we showed that neuroleptics can directly block the motivational differences between the food-sated state and the food-deprived state in the same rats (Experiment 3 ). Taken together, these results suggest that the motivational effects produced by neuroleptics depend wholly on the deprivation state of the animal. They lead to a new model of dopamine's role in motivation that suggests that dopamine neurons mediate motivational effects only when animals are in some type of deprivation state. Indeed, the original results that led to the anhedonia hypothesis, demonstrating that neuroleptics blocked the rewarding effects of food, were made in rats that were deprived of food for 16-20 h (Wise et al., 1978). Our present hypothesis of dopamine's motivational role maintains the most attractive attribute of the anhedonia hypothesis-that one dopamine system is involved in the rewarding effects of a variety of stimuli (e.g., opiates and food). However, it identifies a fracture line in the organization of motivational processes that cuts not between different rewarding stimuli, but along the line defining whether animals are in a deprivation state or not.

Perhaps the most compelling evidence suggesting that motivational processes are divided between those occurring in nondeprivation versus deprivation states comes from brain manipulations that double dissociate the rewarding effects of both opiates and food that occur in nondeprived versus deprived states. Lesions of the TPP (an output of the limbic system in the brainstem) block the rewarding effects of food in nondeprived rats and of opi- 
ates in previously drug-naive rats (not opiate dependent nor in drug withdrawal) (Bechara \& van der Kooy, 1992). However, lesions of the TPP do not block either the aversiveness of morphine withdrawal or the rewarding effect of morphine in opiate-dependent and opiate-withdrawn rats, nor the aversiveness of hunger or the rewarding effects of food in food-deprived rats (Bechara et al., 1992; Bechara \& van der Kooy, 1992). On the other hand, neuroleptics do not block the rewarding effects of opiates in opiate-naive animals (a nondependent and thus nondeprived state), but do block the rewarding effects of opiates in opiate-dependent and opiate-withdrawn animals as well as the aversiveness of withdrawal after the animals are deprived of opiates (Bechara et al., 1992). Similarly, with food, neuroleptics do not block the rewarding effects of food in food-sated rats (Bechara et al., 1992) but do block food reward in food-deprived rats (Wise et al., 1978) as well as the aversiveness of hunger in fooddeprived rats (Bechara et al., in press), which was also found in the present results. This ability of neuroleptics to block both the aversiveness of the deprivation state as well as the rewarding effects of stimuli that overcome the deprivation state leads one to view dopamine neurons as not simply a direct mediator of either the rewarding or aversive aspects of motivation. Rather, dopamine neurons mediate a class of motivational events (regardless of their rewarding or aversive value) that are dependent on an existent state of deprivation.

\section{REFERENCES}

Bechara, A., Harrington, F., Nader, K., \& van der Kooy, D. (1992). Neurobiology of motivation: Double dissociation of two motivational mechanisms mediating opiate reward in drug-naive versus drug-dependent animals. Behavioral Neuroscience, 106(5), 798-807.

BecharA, A., \& van der KoOY, D. (1989). The tegmental pedunculopontine nucleus: A brainstem ouptut of the limbic system critical for psychoactive drug reward. Joumal of Neuroscience, 9, 3400-3409.

BechARA, A., \& VAN DER KOOY, D. (1992). A single brainstem substrate mediates the motivational effects of both opiates and food in non deprived rats but not in deprived rats. Behavioral Neuroscience, 106, 351-363.

Blumberg, J. B., TAYLOR, R. E., \& SUlSER, F. (1975). Blockade of pimozide of a noradrenaline sensitive adenylate cyclase in the limbic forebrain: Possible role of limbic noradrenergic mechanisms in the mode of action of antipsychotics. Joumal of Pharmacy \& Pharmacy \& Pharmacology, 27, 125-128.

Bowman, W. C., RAND, M. J. (1980). Social pharmacology: Drug use for nonmedical purposes: Drug dependence. In W. C. Bowman \& M. J. Rand (Eds.), Textbook of pharmacology (2nd ed., pp. 42.7442.78). Oxford: Blackwell Scientific Publications.

BozArTh, M. A., WISE, R. A. (1981). Heroin reward is dependent on a dopaminergic substrate. Life Science, 29, 1881-1886.

Corbett, D., Stellar, J. R., Stinus, L., Kelley, A., \& Fouriezos, G. (1983). Time course action of alpha-flupenthixol action explains "response artifacts" of neuroleptic action on brain stimulation reward. Science, 222, 1251-1252.

Enna, S. J., Bennett, J. P., Burt, D. R., Creese, I., SNyder, S. H. (1976). Stereospecificity of interaction of neuroleptic drugs with neurotransmitters and correlation with clinical potency. Nature, 263, 338-342.

Etrenberg, A., Pettit, O., Bloom, F. E., \& Кoob, G. F. (1982). Heroin and cocaine intravenous self-administration in rats: Mediation by separate neural systems. Psychopharmacology, 78, 204-209.

Evans, K. R., \&accarino, F. J. (1990). Amphetamine and morphine- induced feeding: Evidence for involvement of reward mechanisms. Neuroscience \& Biobehavioral Reviews, 14, 9-22.

GuICK, S. D., \& Cox, R. D. (1975). Self-administration of haloperidol in rats. Life Science, 16, 1041-1046.

Hand, T. H., Stinus, L., LeMoal, M. (1989). Differential mechanisms in the acquisition and expression of heroin induced place preferences. Psychopharmacology, 98, 61-67.

Hoffman, D. C., \& Beninger, R. J. (1989). The effects of selective dopamine D1 or D2 receptor antagonists on the establishment of agonist induced place conditioning in rats. Pharmacology, Biochemistry \& Behavior, 33, 273-279.

irwin, J., Tombaugh, T. N., Zacharko, R. M., anisman, H. (1983). Alteration of exploration and the response to food associated cues after pimozide treatment. Pharmacology, Biochemistry \& Behavior, 18, 234-246.

MACKEY, B. W., \& VAN DER KOOY, D. (1985). Neuroleptics block the positive reinforcing effects of amphetamine but not of morphine as measured by place conditioning. Pharmacology, Biochemistry \& Behavior, 22, 101-105.

Mithani, S., Martin-Iverson, M. T., Phillifs, A. G., * Fibiger, H. C. (1986). The effects of haloperidol on amphetamine and methylphenidate-induced conditioned place preferences and locomotor activity. Psychopharmacology, 90, 247-252.

Mucha, R. F., IVERSEN, S. D. (1984). Reinforcing properties of morphine and naloxone revealed by conditioned place preference: $A$ procedural examination. Psychopharmacology, 82, 241-247.

Mucha, R. F., van der Kooy, D., O'Shaughnessy, M., BuceNIEKS, P. (1982). Drug reinforcement studies by the use of place conditioning in rat. Brain Research, 243, 91-105.

Rasmussen, K., Beitner-Johnson, D. B., Krystal, J. H., AghajaNIAN, G. K., Nestler, E. J. (1990). Opiate withdrawal and the rat locus coeruleus: Behavioral, electrophysiological and biochemical correlates. Joumal of Neuroscience, 10, 2308-2317.

Redmond, D. E., KRYSTAL, J. H. (1984). Multiple mechanisms of withdrawal from opioid drugs. Annual Review of Neuroscience, 7 , 443-478.

Rowland, N., ENGLE, D. J. (1977). Feeding and drinking interactions after acute butyrophenone administration. Pharmacology, Biochemistry \& Behavior, 7, 295-301.

SALAMONE, J. D. (1992). Complex motor and sensorimotor functions of striatal and accumbens dopamine: Involvement in instrumental behavior processes. Psychopharmacology, 107, 160-174.

SEeman, P. (1980). Brain dopamine receptors. Pharmacological Review, 32(3), 229-313.

Shippenberg, T. S., \& Herz, A. (1987). Place preference conditioning reveals the involvement of D1-dopamine receptors in the motivational properties of $u$ and $k$ opioid agonists. Brain Research, 436, 169-172.

SHIPPENBERG, T. S., \& HeRZ, A. (1988). Motivational effects of opioids: Influence of D-1 versus D-2 receptor antagonists. European Journal of Pharmacology, 151, 233-242.

Spyraki, C., Fibiger, H. C., Phillips, A. G. (1982a). Attenuation by haloperidol of place preference conditioning using food reinforcement. Psychophamacology, 77, 379-382.

Spyraki, C., Fibiger, H. C., \& Phillips, A. G. (1982b). Dopaminergic substrates of amphetamine-induced place preference conditioning. Brain Research, 253, 185-193.

VAN DER KOOY, D. (1987). Place conditioning: A simple and effective method for assessing the motivational properties of drug. In M. A. Bozarth (Ed.), Methods of assessing the reinforcing properties of abused drugs (2nd ed., pp. 229-240). New York: Springer-Verlag.

WISE, R. A., RAPTIS, L. (1986). Effects of naloxone and pimozide on initiation and maintenance measures of free feeding. Brain $R e-$ search, 368, 62-68.

Wise, R. A., Spindler, J., DeWit, H., Gerber, G. J. (1978). Neuroleptic induced "Anhedonia" in rats: Pimozide blocks reward quality of food. Science, 201, 262-264.

YOKEL, R. A., * WISE, R. A. (1976). Attenuation of intravenous amphetamine reinforcement by central dopaminergic blockade in rats. Psychopharmacologia, 48, 311-318.

(Manuscript received May 12, 1992; revision accepted for publication August 25, 1992.) 